\title{
Imaging of Droplet Profiles during Drainage of a Film between a Droplet and an Interface
}

\author{
M.L. Gee' ${ }^{1}$ D.G. Goodall' and G.W. Stevens ${ }^{2}$ \\ 1 Department of Chemistry, University of Melbourne, Parkville 3052, Victoria - Australia \\ 2 Department of Chemical Engineering, University of Melbourne, Parkville 3052, Victoria - Australia \\ e-mail: mlgee@unimelb.edu.au
}

\begin{abstract}
Résumé - Imagerie de profils de gouttes lors du drainage d'un film entre une goutte et une interface - Nous avons utilisé un ellipsomètre/réflectomètre en mode « imager » pour étudier le phénomène de coalescence. Nous avons montré, de manière spécifique, que le film de drainage entre une goutte d'huile déformable et une surface solide peut être étudié par cette technique. Nous en tirons le profil en temps réel de la goutte lors du drainage, lorsque cette goutte s'approche d'une interface. Cette méthode offre l'avantage d'être sensible aux films aussi bien épais que fins. Elle fournit aussi directement un profil du film.
\end{abstract}

Nous avons déterminé la forme d'une goutte d'huile dans un réservoir d'eau lorsque cette goutte approche une surface de silicium. Lors du drainage de cette huile, la goutte se transforme progressivement en film et devient plus plate. L'addition de petites quantités de surfactant déstabilise le film formé, le drainage devient alors non-uniforme jusqu'au point de rupture. Pour une très petite concentration de surfactant, nous avons observé un attachement de la goutte sur la surface, ainsi qu'une rupture incomplète.

Mots-clés : déformation de goutte, drainage de film, ellipsomètre « imager », profil de film, coalescence, rupture, stabilité de film.

\footnotetext{
Abstract - Imaging of Droplet Profiles during Drainage of a Film between a Droplet and an Interface - We have applied imaging ellipsometry/reflectometry to the study of coalescence phenomena. Specifically, we have shown that film drainage between a deformable oil droplet and a solid surface can be monitored using imaging ellipsometry/reflectometry which yields the real time profile of a droplet as film drainage occurs, on approach of the droplet to an interface. The technique has the advantages that it is sensitive to the measurement of both thick and thin films and yields a film profile without the need to scan across the surface.

We have profiled the shape of an oil droplet in a continuous aqueous phase as the droplet approached a silica surface. During film drainage the droplet was observed to dimple, evolving to a flattened profile. The addition of small concentrations of surfactant destabilised the film. Film drainage was non-uniform until the point of film rupture. At very low surfactant concentrations droplet attachment and incomplete rupture were observed.

Keywords: droplet deformation, film drainage, imaging ellipsometry, film profile, coalescence, rupture, film stability.
} 


\section{INTRODUCTION}

This paper describes the use of imaging ellipsometry/ reflectometry for profiling an oil droplet in an aqueous medium as it approaches a solid surface. Our results are applicable to processes that involve liquid/liquid systems such as tertiary oil recovery and liquid-liquid in extraction. When a droplet approaches a surface, such as the approach of two droplets in a liquid/liquid system such as an emulsion, a thin liquid film forms between the drop and the surface [1]. The rate at which this film thins is the rate at which the liquid drains from the gap between the two droplets. When the film becomes sufficiently thin, $\sim 100 \mathrm{~nm}$, the effects of Van der Waals and electrostatic forces become significant. In stable liquid/liquid systems, the film will thin to an equilibrium thickness at which point no further thinning of the intervening fluid between the droplet and the surface occurs. This equilibrium thickness is dependent upon the magnitude of the disjoining pressure in the film which, in this case, acts to stabilise the film. If the system is unstable, the film continues to thin to what is termed the critical thickness, at which point an instability develops, the film ruptures, and the droplet coalesces at the interface.

The shape of a drop as film drainage occurs depends on the deformability of both the droplet and the interface to which it approaches. The extent of deformation is dependent on the viscosity of the continuous phase, interfacial tension, the size of the droplet, and the density difference between the two phases. Earlier work [2, 3] has shown that when an initially spherical droplet approaches a surface, the film between the droplet and the surface drains. However, the rate of drainage of the film is not everywhere uniform. The outer regions of the film are relatively thick due to the spherical shape of the droplet, whereas, the centre of the film is relatively thin since it is the point of closest approach of the droplet to the surface. Therefore, drainage from the periphery of the film, i.e. the barrier ring, is opposed by only a small viscous drag compared to the viscous drag opposing drainage from the thinner, central film area. Consequently, drainage from the barrier ring is initially greater than drainage from the central region of the film and so excess liquid is trapped at the film's centre, thereby deforming the droplet interface into a dimpled shape. Dimpling is particularly pronounced if the droplet is approaching a non-deformable surface. A positive Laplace pressure, resulting from the reverse curvature of the droplet, drives further drainage of the fluid trapped in the central dimple region.

Many investigators have employed the technique of interferometry [4-10] to examine profile changes of a droplet or bubble approaching a surface. Interferometry, although capable of determining droplet profiles with a relatively small degree of error, is restricted to the study of relatively thick films. Compared to other techniques, imaging ellipsometry/reflectometry has the advantage of high vertical resolution, enabling the study of both thick and thin films in real time. Additionally, the entire droplet profile is sampled simultaneously; there is no need to scan across the surface as with certain other techniques that can only give a film thickness at one point at a time. Thus the process of film drainage between a droplet and a surface can be monitored in its entirety until the point at which film rupture occurs.

Imaging ellipsometry has been employed previously to investigate profiles of siloxane oil droplets spreading on glass, silica and mica [11], protein patterning on silica surfaces [12], and domains of surfactant monolayers at an air/water interface [13]. In this paper, we describe the application of imaging ellipsometry/reflectometry to study the system of an oil (squalane) droplet approaching a hydrophilic, negatively charged silica plate in a continuous aqueous medium. We present the effects of varying the ionic strength of the continuous phase and how added surfactant affects the droplet profile. The paper also contains a sample of some recent images of an oil droplet in an aqueous medium approaching a hydrophobed surface.

\section{EXPERIMENTAL SECTION}

\subsection{Ellipsometry/Reflectometry}

Ellipsometry [14] is a polarimetric technique which involves the measurement of changes in polarisation of monochromatic light upon reflection from a film covered surface. Information regarding refractive indices and thicknesses of thin films for a variety of systems can be determined from these changes in polarisation. In a substrate/film/droplet system, such as that of interest in this study, a plane polarised incident beam is reflected and refracted at the substrate/film interface. The portion of the light refracted into the film undergoes reflection at the film/droplet interface and, as a result, suffers a phase change, $\beta$, relative to the light reflected at the substrate/film interface. $\beta$ is related to the thickness of the film, $d$, and the film's refractive index, $n_{2}$, via the expression:

$$
\beta=2 \pi(d / \lambda)\left(n_{1}-n_{2} \sin ^{2} \phi_{1}\right)^{1 / 2}
$$

where $\lambda$ is the wavelength of the incident monochromatic light, $\phi_{1}$ is the angle of incidence and $n_{1}$ is the refractive index of the substrate.

The Fresnel reflection coefficients [15] of the resultant reflected beam are:

$$
\begin{aligned}
& R_{p}=r_{p 12}+r_{p 23} e^{-2 \beta i} / 1+r_{p 12} r_{p 23} e^{-2 \beta i} \\
& R_{s}=r_{s 12}+r_{s 23} e^{-2 \beta i} / 1+r_{s 12} r_{s 23} e^{-2 \beta i}
\end{aligned}
$$

where $r_{12}$ and $r_{23}$ are the Fresnel reflection coefficients at the 1-2 and 2-3 interfaces respectively. 
Experimentally, ellipsometry gives a measure of the ratio of the complex Fresnel reflection coefficients for $p$ and $s$ components as given by Equation (4), viz.:

$$
\rho=R_{p} / R_{s}=\tan \psi e^{-i \Delta}
$$

where tan $\psi=\left|r_{p 12}\right| /\left|r_{s 12}\right|$ and $\Delta=\delta_{r p}-\delta_{r s,} \delta_{r p}$ and $\delta_{r s}$ being the phase shifts experienced upon reflection for the $p$ and $s$ components respectively. Thus, a measurement of $\delta$ for a particular system gives a measure of the thickness of a film of known refractive index.

For the case in which unpolarised light is incident at the substrate/film interface, film thicknesses can be obtained by measuring the intensity of the light reflected from the sample surface. The ratio of the intensity of the reflected light to the intensity of the incident light is commonly known as reflectance, $R$, and is given by:

$$
R=R_{p}+R_{s} / 2
$$

What distinguishes imaging ellipsometry from conventional ellipsometry is that a film thickness profile can be obtained by imaging the reflected intensity over a given area with a CCD (digital) camera and analysing the film thickness spatially. The lateral resolution is therefore defined by the pixel size of the CCD camera. Film thickness resolution is

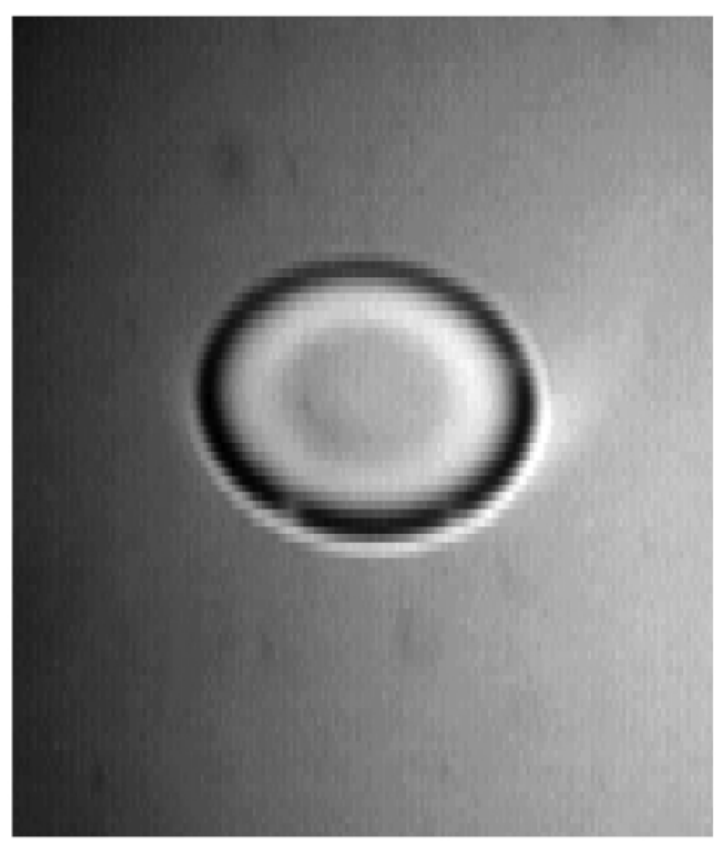

Figure 1

Typical grey-scale image of a droplet approaching a solid surface in a liquid continuous phase. The lighter regions correspond to areas where the film thickness is thick. The image becomes darker the thinner the film. Hence, in this image, the droplet is deformed such that it is dimpled at its centre with the periphery of the droplet, i.e. the barrier ring, the region of closest approach of the droplet to the surface. not compromised. Thus, a grey-scale image is captured. A typical grey-scale image for the system of an oil drop separated from a solid surface by a thin aqueous film is shown in Figure 1. The variation in intensity of the reflected light is indicated by the variation in the grey scale, ranging from white which corresponds to a very thick film, to black which corresponds to a very thin (Newton black) film. This captured grey-scale image clearly illustrates that the droplet has become dimpled at its centre whereas the region around the periphery of the droplet, i.e. the barrier ring, is the part of the droplet closest to the surface.

A modified Beaglehole Instruments imaging ellipsometer was used for all measurements. Details of this microscopic imaging ellipsometer are described elsewhere [16].

\subsection{Experimental Procedure}

Experimentally, a silica prism is placed to seal on top of a glass cylindrical cell filled with the fluid of the continuous phase (aqueous solution) and located at the ellipsometer axis. The silica is the surface to which the oil droplet approaches. Droplets are formed from a stainless steel capillary tip which is positioned $2 \mathrm{~mm}$ from the silica/aqueous interface. The capillary is connected to a gastight syringe contained in a dispenser which provides volume control. The optical components, located on two motorised, rotatable arms, are arranged for reflection from the silica/aqueous interface. In a typical experiment, a droplet is rapidly expanded from the capillary tip. Ellipsometric measurements are taken to determine the profile of a droplet as it approaches the silica surface and drainage of the aqueous film between the droplet and the surface occurs. The time resolution for these experiments is about $1 \mathrm{~s}$, which allows for accurate monitoring of the changes in the profile of the droplet as it continually approaches the surface.

Solution conditions for all experiments were set such that the silica plate had an overall negative charge. Thus for all experiments, the aqueous phase was set to a $\mathrm{pH}$ of 6.8 using a dilute $\mathrm{NaOH}$ solution. Droplet profiles and film drainage were hence monitored at this $\mathrm{pH}$ but as a function of electrolyte concentration $\left(10^{-3}, 10^{-4}\right.$ and $\left.10^{-5} \mathrm{M} \mathrm{NaCl}\right)$.

Film drainage was also monitored in the presence of added surfactant $\left(10^{-3} \mathrm{M}\right.$ cetyltrimethylammonium chloride, CTAB) at $\mathrm{pH} 6.8$ and $10^{-3} \mathrm{M}$ backround salt. In one set of surfactant experiments, the silica plate was rendered hydrophobic by deposition of a cross-linked fluorocarbon film and film drainage monitored [17].

\subsection{Materials}

The two immiscible fluids used in these experiments were squalene (droplet phase), and water (continuous phase). Squalene $\left(\mathrm{C}_{30} \mathrm{H}_{50}\right)$ was obtained from Sigma Aldrich 
Chemical Co. Pty. Ltd. (98+\%) and was purified by vacuum distillation. The water used in the experiments was produced using a Milli-Q-filtration system. Sodium chloride was obtained from Ajax Chemicals (99.9+\%). The surfactant, CTAB, was obtained from Eastman Kodak (Rochester, NY) and was further purified by twice recrystallising from an ethanol/acetone mixture.

The equilateral dispersing prism made of fused silica was supplied by Ealing optics. The prism was removed of impurities firstly by light scrubbing with a warm solution of Extran 100, followed by thorough rinsing with Milli-Q water. The prism was then further treated with a hot ammoniacal peroxide solution ( 2 parts hydrogen peroxide, 1 part water, 1/10 ammonia) before copious rinsings with Milli-Q water.

The capillary was soaked in Extran 100 for about $12 \mathrm{~h}$, rinsed with Milli-Q water before being emersed in a hydrofluoric acid wash $(60 \%$ water, $35 \%$ conc. nitric acid, $5 \%$ hydrofluoric acid), followed be repeated rinsings with Milli-Q water.

The syringe casing was washed in an Extran solution $(\sim 2 \mathrm{~h})$, nitric acid washed $(\sim 12 \mathrm{~h})$ before numerous rinsings with Milli-Q water. All other glassware and fittings were scrubbed and soaked in an Extran solution for about $12 \mathrm{~h}$, rinsed with Milli-Q water, then soaked in concentrated base $(\mathrm{NaOH})$. The glassware and fitting were then finally rinsed numerous times with Milli-Q water.

\section{RESULTS AND DISCUSSION}

\subsection{Film Drainage as a Function of Salt Concentration}

Typically, after the approach of a droplet towards the silica plate, the droplet initially deforms into a dimple type shape, as illustrated in Figure 1. Droplet profiles are obtained by taking a cross-section through the grey-scale image and determining the ellipsometric thickness of the film between the droplet and the surface along this cross-section. Figure 2 contains a set of such droplet profiles for the system of a squalene droplet expanded towards a hydrophilic, negatively charged silica plate in a continuous medium of $10^{-3} \mathrm{M} \mathrm{NaCl}$ at $\mathrm{pH}$ 6.8. Each profile corresponds to a particular time in the film drainage process and so we monitor the evolution of the droplet shape as film drainage occurs. Note that during the course of an experiment, several more profiles are obtained but only a few are shown in Figure 2 for clarity.

The droplet profiles in Figure 2 for $10^{-3} \mathrm{M} \mathrm{NaCl}$ illustrate the dimpled profile of the droplet because of the hindered drainage of the film at the droplet's centre, as detailed above. Once the film thickness at the barrier ring is near its equilibrium thickness, drainage from the central film area continues allowing the shape of the droplet to continually evolve from a dimpled to a flattened profile. Similar drainage

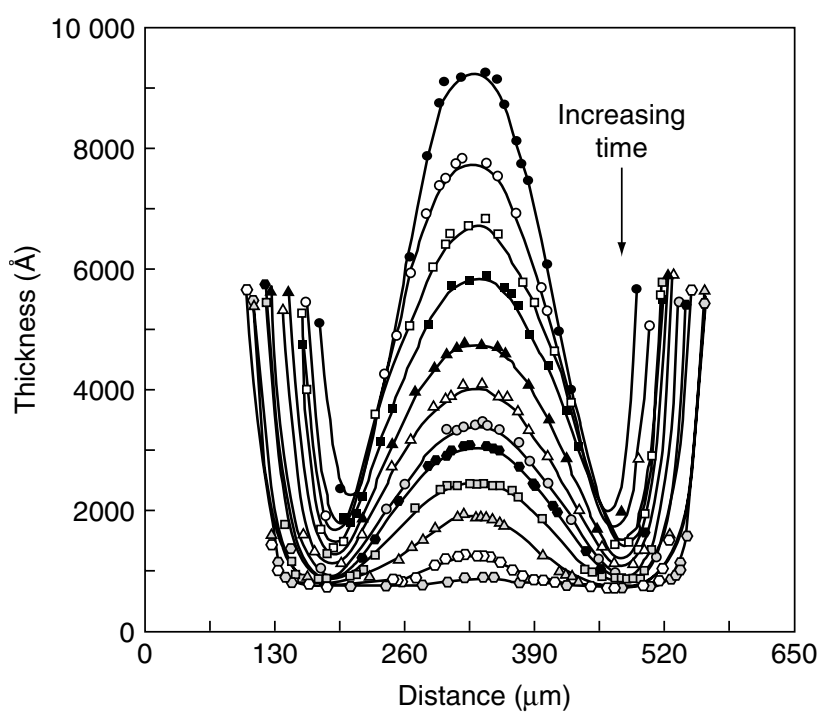

Figure 2

Droplet profiles for the system of a squalene droplet approaching a hydrophilic silica plate in a continuous medium of $10^{-3} \mathrm{M} \mathrm{NaCl}(\mathrm{pH}=6.8)$ for a range of times during film drainage. These plots of film thickness versus lateral distance across the droplet are obtained by taking a cross-section through a grey-scale image of a droplet approaching a surface and determining the film thickness along this cross-section. Profiles obtained in this manner show the evolution of the droplet shape as film drainage occurs and show the film dimple and then flatten to a stable equilibrium film.

behaviour is also exhibited under conditions of lower salt concentrations $\left(10^{-4} \mathrm{M}\right.$ and $\left.10^{-5} \mathrm{M} \mathrm{NaCl}\right)$. The droplet dimples on approach to the silica plate and the system comes to equilibrium such that the droplet is flattened and an aqueous film is stabilised between the droplet and the silica plate.

It has been reported $[18,19]$ that organic droplets present in aqueous solution show similar characteristics to air bubbles in an aqueous environment and bear a net negative charge due to preferential adsorption of ions at the oil/aqueous interface. Consequently, as an oil droplet approaches a similarly charged plate, electrical double layer repulsion exists between the droplet and surface hindering film drainage. When the pressure inside the film is equal to the pressure inside the droplet, no further film drainage occurs and an equilibrium film of uniform thickness results.

Figure 3 shows the rate of drainage data, i.e. film thickness at the barrier ring as a function of time, obtained for the range of salt concentrations investigated in this study. Note that the $y$-axis is plotted on a $\log$ scale. For each drainage curve there are two distinct linear regions, for convenience named Region 1 and Region 2. This is indicative of a specific drainage rate constant for each region 
where the rate constant is proportional to the gradient. In the initial stages of drainage (Region 1), the rate constant is significantly larger than that in Region 2. Drainage eventually ceases and a stable aqueous film is formed.

It can be seen from Figure 3 when comparing the gradients of the log (film thickness) versus time curves, that, in Region 1 (initial stages of drainage), film drainage is more rapid the higher the salt concentration. The factors affecting film drainage are the interfacial tension and density differences between the oil droplet and the continuous aqueous phase, the viscosities of the oil and the aqueous solution, and the distance between the droplet and the silica surface. In our system, we have measured the interfacial tension and viscosities and found that they are independent of salt concentration. The only variable in our system is salt concentration and the only thing that this affects is the Debye length. Hence it follows that the explanation for the difference in drainage rate must be the variation in the range of the double layer repulsion between the droplet and the silica surface. The lower the double layer repulsion the less the resistance to the approach of the droplet to the surface [6]. This leads to a reduction in drainage rate since the droplet and silica surface are closer together hindering flow of the aqueous phase from between the droplet and the surface.

Another feature of Figure 3 is the film thickness where the transition between Regions 1 and 2 occurs. This transition point increases the lower the salt concentration, as shown in Table 1. This observation is also a result of the double layer repulsion between the droplet and the silica surface hindering the approach of the droplet under low salt conditions.

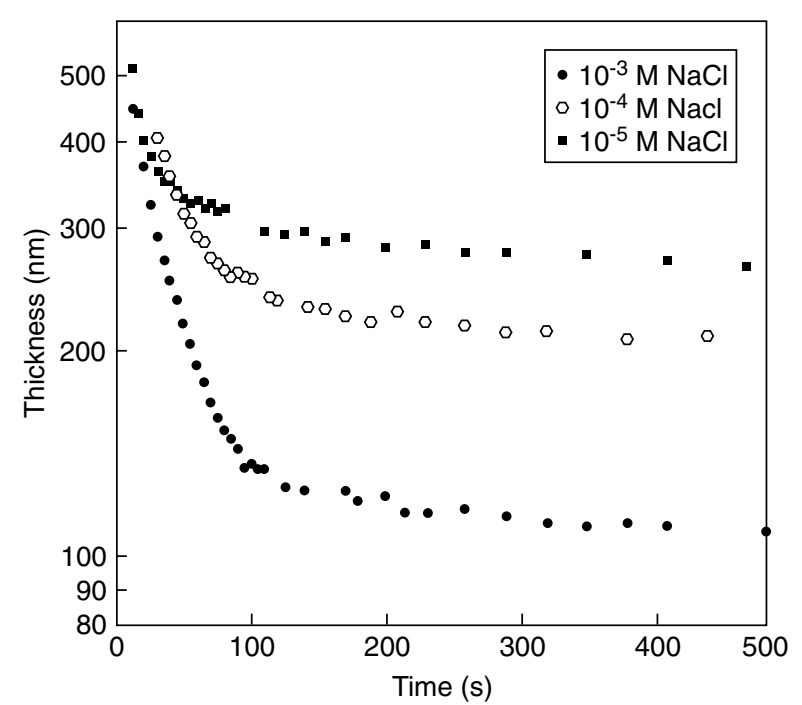

Figure 3

Semi-log plot of film thickness at the barrier ring as a function of time for the systems of a squalene droplet approaching a hydrophilic silica plate in an aqueous continuous phase.
TABLE 1

Transition point versus salt concentration

\begin{tabular}{c|c|c}
\hline $\begin{array}{c}\mathrm{NaCl} \text { concentration } \\
(\mathrm{M})\end{array}$ & $\begin{array}{c}\text { Transition point } \\
(\mathrm{nm})\end{array}$ & $\begin{array}{c}\text { Equilibrium film thickness } \\
(\mathrm{nm})\end{array}$ \\
\hline $1 \times 10^{-3}$ & 128 & 88 \\
$1 \times 10^{-4}$ & 239 & 145 \\
$1 \times 10^{-5}$ & 339 & 236 \\
\hline
\end{tabular}

Region 2 of the drainage data in Figure 3 is independent of salt concentration, as can be seen when comparing the gradients of the drainage rate curves. Beyond the transition point, drainage becomes hindered and is only very limited. It appears that once the transition point is reached, determined by the range of the double layer repulsion, this repulsion hinders further drainage. The limited amount of drainage that does occur is driven by the Laplace pressure due to the curvature of the droplet. Drainage continues until the droplet flattens and an equilibrium film exists between the droplet and the silica surface. The thicknesses of these equilibrium films as a function of salt concentration are given in Table 1. It is clear from this data that double layer repulsion determines the thickness of the equilibrium film, this thickness increasing when salt concentration is reduced.

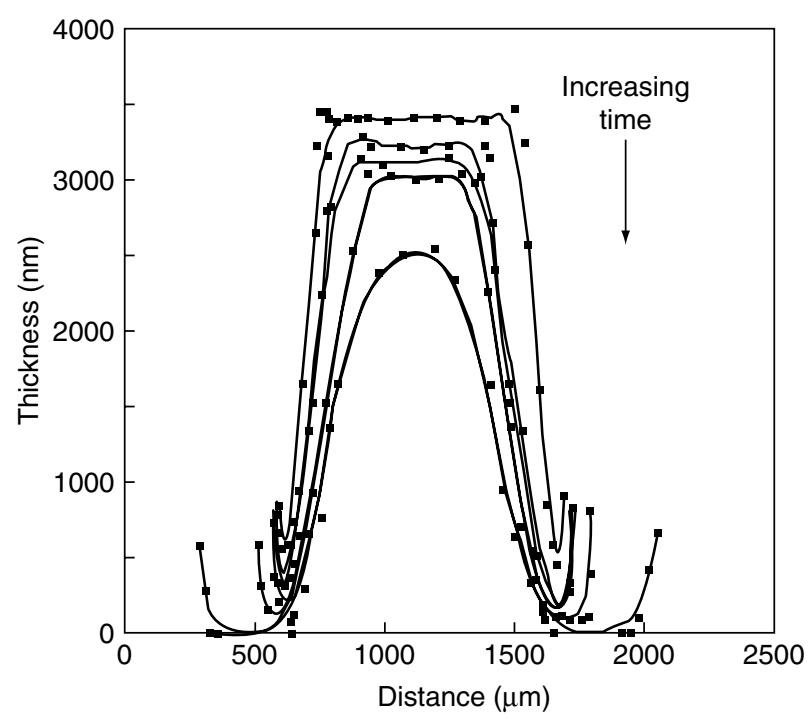

Figure 4

Droplet profiles for the system of a squalene droplet approaching a hydrophilic silica plate in a continuous medium of $10^{-3} \mathrm{M} \mathrm{NaCl}$ with $10^{-7} \mathrm{M} \mathrm{CTAB}$ at $\mathrm{pH}=6.8$ for a range of times during film drainage, similar to Figure 3. Profiles show the evolution of the droplet shape as film drainage occurs and again illustrate dimpling of the droplet in the initial stages of film drainage. During drainage, rupture occurs only at the barrier ring and a quasi-equilibrium is reached where water is trapped in the dimple. 
The data in Figure 4 also indicates that there is a dependence on salt concentration for the time required for the barrier ring to near its equilibrium thickness. In $10^{-3} \mathrm{M}$ salt, this time is about $300 \mathrm{~s}$, in $10^{-4} \mathrm{M}$ salt it takes $1000 \mathrm{~s}$ and in $10^{-5} \mathrm{M}$ salt it takes $2700 \mathrm{~s}$. This is clearly a double layer effect. At higher salt concentrations, the double layer repulsion is reduced allowing faster rate of approach of the droplet to the surface and hence the system moves more rapidly to equilibrium.

\subsection{The Effect of Surfactant on Film Drainage}

The system discussed above is stable in the sense that film drainage between the plate and the droplet continues only until the thickness of the film reaches some equilibrium value. Film rupture does not occur due to the repulsive double layer forces between the plate and the droplet acting to stabilise the film. This is not the case when surfactant is added to the aqueous continuous phase. Droplet profiles for $10^{-3} \mathrm{M} \mathrm{NaCl}$ with $10^{-7} \mathrm{M}$ added CTAB illustrate the effect of added surfactant even when the surfactant concentration is trace. The profiles clearly illustrate the dimpling of the droplet, as seen in Figure 2 when no CTAB is present. However, note that an equilibrium film is not achieved. Instead, film rupture occurs at the barrier ring, at which point the droplet coalesces at the silica surface. In fact, the entire drainage process takes only tens of seconds, as compared to thousands of seconds in the absence of surfactant. When the $\mathrm{CTAB}$ concentration is further increased to $10^{-4} \mathrm{M}$ (Fig. 5), film rupture and coalescence occur in only $10 \mathrm{~s}$.

When an ionic surfactant such as the positively charged CTAB is added to the system, the surfactant will reside at the liquid-liquid interface with the hydrocarbon tails partitioned in the interior of the oil droplet and the polar head groups in the aqueous continuous phase. This will reduce the net charge at the liquid/liquid interface and, at a high enough surfactant concentration, result in charge reversal and a possible attraction between the droplet and the surface [20]. The positively charged CTAB head groups are also attracted to negative sites at the silica surface reducing its net negative charge. The reduction in charge at both interfaces limits the double layer repulsion and thereby increases the rate of film drainage.

We observe film rupture when surfactant is added to our system even at very low surfactant concentrations indicating not only a reduction in double layer repulsion, but also an attraction between the oil droplet and the silica surface. We can best explain how this comes about by considering the equilibrium position of the system. Surfactant is attracted to both the oil droplet and the silica surface, as detailed above. However, the most favourable situation, thermodynamically, is when the surfactant is removed from aqueous solution, driven by the hydrophobic effect, and electroneutral. This can be achieved in the system if, as film drainage occurs,

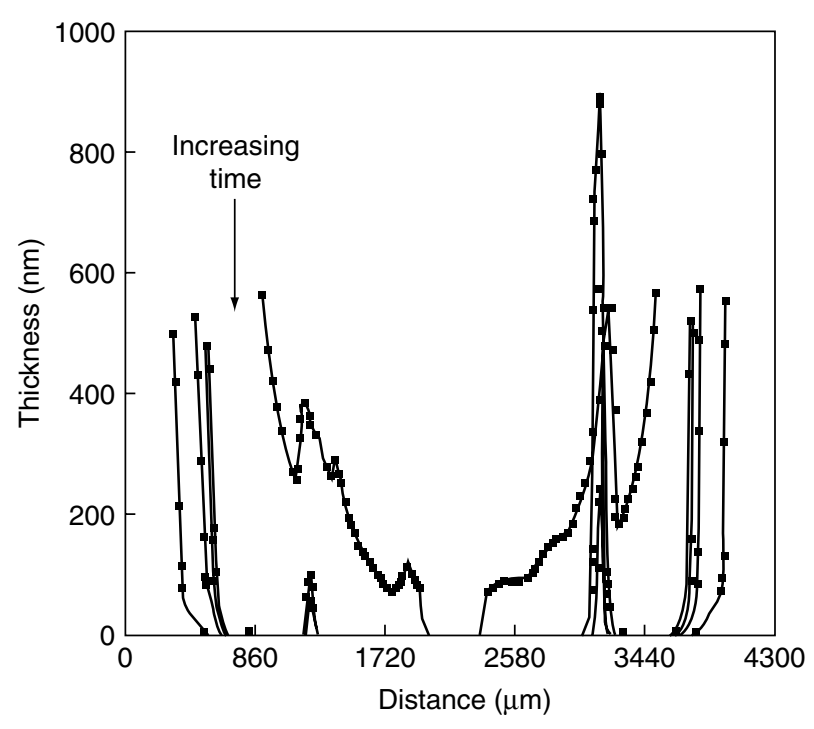

Figure 5

Droplet profiles for the system of a squalene droplet approaching a hydrophilic silica plate in a continuous medium of $10^{-3} \mathrm{M} \mathrm{NaCl}$ with $10^{-4} \mathrm{M} \mathrm{CTAB}$ at $\mathrm{pH}=6.8$ for a range of times during film drainage, similar to Figure 3. Profiles show the evolution of the droplet shape as film drainage occurs and illustrate the non-uniformity of film drainage due to the low interfacial tension. The film ruptures and the droplet coalesces at the surface. The spikes in the profile are narrow channels of water via which film drainage continues until complete flattening of the droplet at the surface.

surfactant at the liquid/liquid interface migrates to the points of closest approach of the droplet to the surface with its positive head groups orientated towards negative sites at the silica surface. The equilibrium situation is that of coalescence of the droplet at the silica surface with the surfactant tails partitioned into the oil droplet and the head groups adsorbed at the silica surface.

The above process occurs at both $10^{-7} \mathrm{M}$ and $10^{-4} \mathrm{M}$ CTAB. There are, however, obvious differences between the profiles during film drainage at both these CTAB concentrations. At $10^{-7} \mathrm{M} \mathrm{CTAB}$, film rupture is observed to occur only at the barrier ring. Once the barrier ring has coalesced at the silica surface, it can be seen from Figure 4 that the droplet spreads over this region. This entire process takes approximately $40 \mathrm{~s}$. Interestingly, some of the aqueous film remains trapped between the surface and the droplet at its centre and so the droplet maintains its dimpled shape. No film drainage was observed from the dimple. The droplet maintained its dimpled shape over several days of observation. This is some sort of quasi-equilibrium state. The Laplace pressure that should lead to further film drainage is somehow prohibited maintaining an unusually large separation $(2000 \AA)$ between the surface and the centre of the droplet. We propose that the reason for this observation is 
that as the droplet approaches the silica surface, CTAB migrates at the liquid/liquid interface to the barrier ring since this is the region closest to the silica surface. There is insufficient CTAB at other points along the liquid/liquid interface to result in complete film drainage, and so the dimple is trapped and only the barrier ring coalesces at the silica surface.

When the CTAB concentration is raised to $10^{-4} \mathrm{M}$, coalescence is complete and film drainage is very rapid, taking only $10 \mathrm{~s}$. At this CTAB concentration, the barrier ring is saturated with surfactant and there is a significant amount of surfactant in the central region of the droplet to facilitate film drainage and coalescence. Note that the increased surfactant concentration reduces the interfacial tension between the droplet and the continuous phase. Consequently, the liquid/liquid interface is not well defined (Fig. 5). Note that as the droplet coalesces at the surface, some spikes appear in the droplet profile. These spikes correspond to small channels where water is transiently trapped between

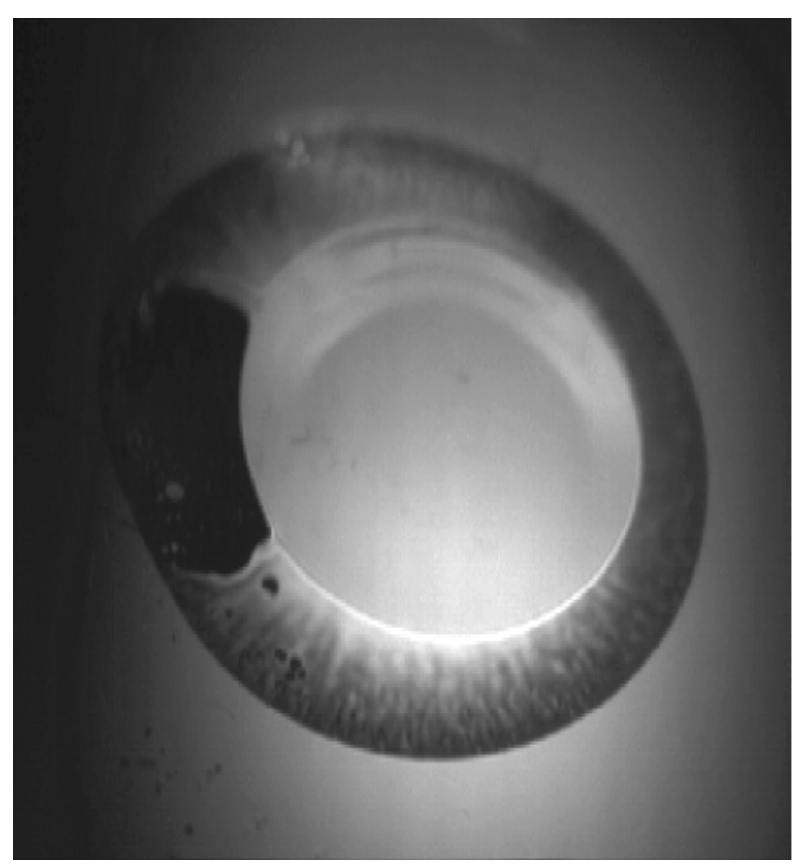

Figure 6

Grey-scale image of a squalene droplet approaching a hydrophobic silica plate in a continuous medium of $10^{-3} \mathrm{M}$ $\mathrm{NaCl}$ with $10^{-3} \mathrm{M} \mathrm{CTAB}$ at $\mathrm{pH}=6.8$. The image illustrates the dimpling of the droplet and shows that the barrier ring is flattened and is the closest region of the droplet to the surface. The narrow channels present in the droplet profiles shown in Figure 5 are clearly visible in this image and appear at light dendritic channels originating at the dimple and extending through the barrier ring towards the bulk continuous phase. the droplet and the surface. These channels are the means by which the remaining water is able to drain until, at equilibrium, the droplet is completely flat over its region of contact with the silica surface.

We have also observed such film drainage channels when an oil droplet approaches a hydrophobed silica surface in the presence of CTAB at $10^{-3} \mathrm{M}$. Figure 6 is one of the grey-scale images captured during film drainage in this system. The dark periphery of the image corresponds to the barrier ring of the droplet that is clearly closer to the surface than the central region of the droplet, i.e. the droplet is dimpled. Note also that the barrier ring is flattened as a result of initial rapid film drainage in this region. There is a very dark portion of the barrier ring at its left hand side indicating that the film between the barrier ring of the droplet and the surface is very thin there. This dark region was observed to spread as film drainage continued. Across the barrier ring there are channels where the reflected intensity is brighter indicating that the aqueous film is relatively thick along the paths of these channels. The channels appear almost dendritic. They originate from the dimple and were observed to fluctuate in intensity slightly when observing the image in real time. Liquid drained from the dimple by means of the dendritic channels until eventually, at equilibrium, the grey-scale image was uniformly black since film drainage was complete.

\section{CONCLUSIONS}

This paper shows that the technique of imaging ellipsometry/ reflectometry is capable of following the evolution of droplet profiles as a function of time as a droplet approaches an interface. To illustrate the usefulness of this technique, we studied the system consisting of a squalene drop approaching a silica surface in a continuous aqueous phase at $\mathrm{pH} 6.8$. We have monitored dimpling of the drop as a result of nonuniform film drainage and have shown that at equilibrium, the droplet flattens such that the film is of uniform thickness. This equilibrium thickness is governed by the double layer interaction between the drop and the silica surface. When surfactant is present, the droplet coalesces at the silica surface due to the migration of surfactant at the liquid/liquid interface so that it resides at the oil/silica surface. When droplet coalescence occurs, film drainage was observed to occur via dendritic water channels running from the centre of the droplet at its dimple through the barrier ring out to the continuous aqueous phase. The results presented above will appear in more detail in future publications.

\section{ACKNOWLEDGMENTS}

The authors gratefully acknowledge the financial support of the Australian Food Industry Science Centre and the Australian Research Council. 


\section{REFERENCES}

1 Jeffreys, G.V. and Davies, G.A. (1971) Recent Studies in Liquid-Liquid Extraction, Pergamon Press, Oxford.

2 Allan, R.S., Charles, G.E. and Mason, S.G. (1961) J. Colloid Sci., 16, 150.

3 Platikanov, D.J. (1964) Phys. Chem., 68, 3619.

4 Burrill, K.A. and Woods, D.R. (1969) J. Colloid Interface Sci., 30, 511.

5 Fisher, L.R., Mitchell, E.E., Hewitt, D., Ralston, J. and Wolfe, J. (1991) Colloids and Surfaces, 52, 163.

6 Burrill, K.A. and Woods, D.R.J. (1973) Colloid Interface Sci., 42, 15.

7 Horn, R.G., Bachmann, D.J., Conner, J.N. and Miklavcic, S.J. (1996) Langmuir, 12, 4197.

8 Aveyard, R., Binks, B.P., Cho, W.G., Fisher, L.R., Fletcher, P.D.I. and Klinkhammer, F. (1996) Langmuir, 12, 6561.

9 Joye, J.L. and Miller, C.A. (1992) Langmuir, 8, 3083.

10 Hewitt, D., Fornasiero, D. and Ralston, J.J. (1993) Chem. Soc. Faraday Trans., 89, 817.
11 Beaglehole, D.J. (1989) Phys. Chem., 93, 893.

12 Reiter, R., Motschmann, H., Orendi, H., Nemetz, A. and Knoll, W. (1992) Langmuir, 8, 1784.

13 Jin, C., Jansson, K. and Arwin, H. (1996) Rev. Sci. Instrum., 8, 67.

14 Azzam, R.M.A. and Bashara, N.M. (1977) Ellipsometry and Polarised Light, Elsevier North-Holland Inc., New York.

15 Born, M. and Wolf, E. (1990) Principles of Optics, Pergamon, Oxford.

16 Beaglehole, D. (1988) Rev. Sci. Instrum., 12, 59.

17 Parker, J.L., Claesson, P.M. and Attard, P.J. (1938) Phys. Chem., 34, 300.

18 Dickinson, W.J. (1941) Chem. Soc. Faraday Trans., 37, 140.

19 Lin, K.L. and Osseo-Asare, K. (1986) Recent Developments in Separation Science, CRC Press.

$20 \mathrm{Gu}$, Y. and Dongquing, L. (1998) Colloids and Surfaces, 139, 213.

Final manuscript received in September 2000 